\title{
Mapeo de actores en la Costa Caribe norte de Nicaragua: un análisis según el género y la identificación étnica y cultural*
}

Stakeholder mapping on the northern Caribbean coast of Nicaragua: an analysis by gender and ethnic and cultural identification

Julie Cupples ${ }^{\text {a }}$

University of Edinburgh, Escocia

julie.cupples@ed.ac.uk

ORCID: https://orcid.org/0000-0002-6126-6190

Recibido: 15 Junio 2018

Guillermo Incer Medina

Aceptado: 18 Diciembre 2020

Unidad Nacional Azul y Blanco, Nicaragua

Publicado: 20 Diciembre 2020

ORCID: https://orcid.org/0000-0001-5640-618X

Irving Larios

Instituto de Investigaciones y Gestión Social (Inges),

Nicaragua

ORCID: https://orcid.org/0000-0002-2726-030X

Dixie Lee Smith

Universidad de las Regiones Autónomas de la Costa Caribe

de Nicaragua (Uraccan), Nicaragua

ORCID: https://orcid.org/0000-0002-9592-4585

\section{Resumen:}

Este artículo se deriva de un estudio en dos municipios de la Costa Caribe norte de Nicaragua, que interactúa de manera crítica con la agenda de investigación británica, dominante en este momento. Mediante un stakeholder analysis, hemos intentado mapear el poder cultural en esta parte de Nicaragua en un contexto de racismo persistente, desigualdad de género, pobreza, destrucción ambiental y creciente autoritarismo político mediante entrevistas, grupos focales y talleres con actores relevantes. La investigación fue realizada por un grupo de académicos, profesionales de una organización no gubernamental (ONG) y activistas de la sociedad civil que viven en la costa o que tienen una participación política y profesional a largo plazo, en la región. Proporciona un conjunto de recomendaciones para los organismos gubernamentales y las organizaciones de la sociedad civil, con potencial de aumentar el impacto positivo de nuestros proyectos de investigación y desarrollo en la región.

Palabras clave: Nicaragua, Costa Caribe, Miskitos, afrodescendientes, género, racismo.

\section{Abstract:}

This article derives from a study in two municipalities on the northern Caribbean coast of Nicaragua, which interacts critically with the currently dominant British research agenda. Through stakeholder analysis, we have attempted to map cultural power in this part of Nicaragua in a context of persistent racism, gender inequality, poverty, environmental destruction and increasing political authoritarianism through interviews, focus groups and workshops with relevant actors. The research was conducted by a group of academics, non-governmental organization (NGO) practitioners and civil society activists living on the coast or with long-term political and professional involvement in the region. It provides a set of recommendations for government agencies and civil society organizations, with potential to increase the positive impact of our research and development projects in the region.

Keywords: Nicaragua, Caribbean coast, Miskitos, Afro-descendants, gender, racism.

\section{Resumo:}

Este artigo é derivado de um estudo em dois municípios da beira-mar caribenha do norte da Nicarágua, que interage de forma crítica com a agenda de pesquisa britânica, dominante no momento. Por meio da stakeholder analysis tentamos mapear o poder

Notas de autor

\footnotetext{
${ }^{\text {a } C o r r e o ~ d e ~ c o r r e s p o n d e n c i a: ~ j u l i e . c u p p l e s @ e d . a c . u k ~}$
} 
cultural nesta parte da Nicarágua em um contexto de racismo persistente, desigualdade de gênero, pobreza, destruição ambiental e crescente autoritarismo político, por meio de entrevistas, grupos focais e workshops com atores relevantes. A pesquisa foi realizada por um grupo de acadêmicos, profissionais de uma organização não governamental (ONG) e ativistas da sociedade civil residentes no litoral ou com longa participação política e profissional na região. Ela fornece um conjunto de recomendações para agências governamentais e organizações da sociedade civil, com potencial para aumentar o impacto positivo de nossos projetos de pesquisa e desenvolvimento na região.

Palavras-chave: Nicarágua, costa caribenha, Miskitos, afrodescendentes, gênero, racismo.

\section{Introducción}

Este proyecto se enfoca en el tema del poder en la Costa Caribe norte de Nicaragua y nace de una colaboración a largo plazo entre la Universidad de Edimburgo, la Universidad de las Regiones Autónomas de la Costa Caribe Nicaragüense (Uraccan), el Instituto de Investigaciones y Gestión Social (Inges) y varias organizaciones de la sociedad civil. La investigación fue financiada por el ESRCIAA (Economic and Social Research Council Impact Accelerator Account) del Reino Unido, un fondo establecido para aumentar el impacto positivo de los proyectos de investigación, y llevada a cabo por cuatro investigadores: tres nicaragüenses y una extranjera que trabajan en el sector académico y en el sector ONG y la sociedad civil.

La Costa Caribe de Nicaragua es una región multiétnica y multicultural, habitada por seis grupos indígenas o étnicos: Miskitos, Mayangnas, Ramas, Creoles, Garífunas y mestizos, colectivamente llamados costeños. La Costa Caribe nunca fue colonizada por España, pero fue anexada de forma violenta por Nicaragua en 1894, un hecho histórico que fue fuente del resentimiento colectivo en la zona. Actualmente, es una región que se enfrenta a una serie de desafíos multifacéticos de carácter social, económico, político, cultural, infraestructural y ambiental. Desde 1987, se reconoció su autonomía política en un marco legislativo bastante amplio. Este marco representa uno de los mecanismos principales que tiene la población costeña para mejorar el nivel de bienestar de los habitantes de la región.

Nuestro trabajo colaborativo está enfocado en los desafíos políticos, sociales, culturales y ambientales a que se enfrenta la Costa Caribe norte de Nicaragua, y en la construcción de la ciudadanía cultural y política. A pesar de tener muchas ventajas estratégicas y mucha riqueza natural y cultural, esta región se caracteriza por altos niveles de vulnerabilidad ambiental y socioeconómica, por abandono estatal con respecto a la inversión en infraestructura y servicios sociales, y por la exposición a los efectos negativos del narcotráfico. Además, dada la realidad multicultural de la región, hay mucho énfasis en el tema de la interculturalidad entre los grupos de la sociedad civil, los medios de comunicación, las agencias del Estado y los intelectuales.

Muchos ciudadanos de la región sienten que la distribución desigual de poder económico, político y cultural entre los pueblos que la conforman, combinada con la desigualdad de género, bastante arraigada, constituyen un obstáculo en cuanto al fortalecimiento de la autonomía y a la participación positiva en los procesos de desarrollo. Especialmente, los grupos indígenas y afrodescendientes, y las mujeres se encuentran marginados en muchos espacios sociales y políticos. La exclusión reduce la efectividad de las intervenciones y debilita las capacidades locales de acabar con las desigualdades económicas y la vulnerabilidad ambiental. Cómo ha notado González (2013, p. 12) en un estudio sobre participación política en Yatama en la Costa Caribe, la sobrerrepresentación de los grupos mayoritarios es percibida por los grupos minoritarios como un efecto negativo de la autonomía, que disminuye su poder de decisión en aspectos importantes acerca de la calidad de vida. Es necesario democratizar el acceso a los espacios de toma de decisión en la Costa Caribe para poder promover una representación más equitativa. En este escenario, creíamos que el impacto de nuestro trabajo podría ampliarse, si tuviéramos un entendimiento más sistemático y detallado de las desigualdades existentes en cuanto al género e identidades culturales.

Bajo el objetivo propuesto, decidimos llevar a cabo un análisis de actores (stakeholder analysis) mediante el cual identificamos y mapeamos el poder económico social, político y cultural en la región según género e 
identidad cultural y étnica en dos municipios de la costa Caribe norte: Bilwi o Puerto Cabezas y Waspam. Rastreamos y documentamos a las personas que ocupan posiciones de poder en los gobiernos municipales, regionales y territoriales; la Alcaldía municipal; las ONG; los movimientos sociales y partidos políticos de la región; los medios de comunicación; las iglesias, y en los centros de comercio. Mediante la participación en grupos focales y reuniones en los cuales divulgamos los resultados preliminares, los ciudadanos costeños tuvieron la oportunidad de expresar sus opiniones acerca de su acceso a los espacios de toma de decisión. Entendemos por actores todas las personas que inciden mediante su trabajo o su activismo en la vida cultural, social o política de la costa Caribe.

Esperamos que este estudio ayude a las autoridades del Gobierno, las ONG y las organizaciones de la sociedad civil a dirigir recursos, de forma más eficiente, a los grupos menos representados y fomentar la participación de estos. Asimismo, proyectamos que este ejercicio investigativo se convierta en una herramienta utilizada por estas entidades en el momento de hacer nombramientos en cargos remunerados y no remunerados, convocar a talleres, simposios o capacitaciones, y elaborar políticas. Además, planteamos que sea de utilidad para los grupos menos representados, como las mujeres indígenas, para mejorar su posición social. En conjunto, esperamos que esta investigación posibilite el equilibrio de poder y transforme las prácticas de marginación de importantes sectores y grupos. En ese sentido, buscamos el empoderamiento de los actores existentes y la identificación de nuevos actores con capacidad de incidencia en la región.

\section{Enfoque metodológico y métodos seguidos}

La principal metodología del proyecto fue el stakeholder analysis o un análisis de actores (Tapella, 2007; Almada Navarro, 2009), basado en el análisis decolonial, que enfatiza en los conocimientos populares e indígenas, define el colonialismo, el capitalismo y el patriarcado como elementos que se constituyen mutuamente y toma una posición crítica con respeto al desarrollo convencional. Este reconoce que, aunque Nicaragua se convirtió en país independiente en el siglo XIX, las actitudes y las prácticas coloniales no fueron eliminadas, al contrario, dieron lugar a una nueva colonialidad, sistema que sigue existiendo.

El trabajo se dividió en siete fases principales: trabajo de campo y recopilación de información, divulgación de resultados preliminares en dos talleres en Bilwi y Managua, elaboración del documento escrito, difusión del texto entre los actores principales en la costa y en Managua e invitación de comentarios o reflexiones, revisión del documento a partir de los comentarios y aportes recibidos, y publicación del artículo final en una revista académica de acceso abierto.

Este enfoque metodológico contempla el reconocimiento y la inclusión de las voces y perspectivas de muchas personas en el artículo, quienes han tenido varias oportunidades de contribuir al proceso. También significa que se trata de un estudio que procura hablar a diversos públicos. Aunque la Costa Caribe es una región donde se hablan varios idiomas, entre estos el miskitu, el mayagna, el inglés creole y el español, la publicación de este artículo en español permite divulgación ante una mayor cantidad de lectores.

El trabajo de campo consistió en la ejecución de seis grupos focales y dos entrevistas semiestructuradas de aproximadamente una hora, ambos espacios grabados, que se desarrollaron en septiembre de 2016, en los municipios de Bilwi y Waspam. Representantes de todos los pueblos y comunidades de la región fueron invitados a participar en los grupos focales, donde tuvieron la oportunidad de dar su perspectiva y tocar estos temas de manera profunda. Los invitados fueron hombres y mujeres que tenían cargos en gobiernos regionales, municipales o territoriales, eran líderes de ONG o movimientos sociales, dirigían o tenían puestos en periódicos o canales de radio o televisión, eran intelectuales o empresarios destacados, y trabajaban como pastores o líderes religiosos en las principales iglesias de la región. Al convocar a los participantes, se invitó a miembros de todos los grupos indígenas y comunidades étnicas que viven en la Costa Caribe, también intervinieron mujeres y hombres. Para cada grupo, completamos una plantilla para 
establecer quienes ya ocupaban posiciones de poder. Había una serie de preguntas guía de estos grupos focales (tabla 1), que, sin embargo, eran flexibles, es decir, los participantes podían poner sobre la mesa otras perspectivas propias, siempre y cuando, estuvieran tratando los temas centrales del proyecto. Identificamos los siguientes temas como centrales para el proyecto: poder, representación, género, identidad cultural, espacios de toma de decisión y proceso de autonomía; participación en la política, el proceso autonómico y los procesos de desarrollo; voz y posibilidad de expresarse libremente; racismo; machismo; discriminación; interculturalidad; mestizaje, y flujos migratorios. Estos produjeron discusiones muy animadas en los grupos focales y las entrevistas.

TABLA 1.

Esquema para grupos focales

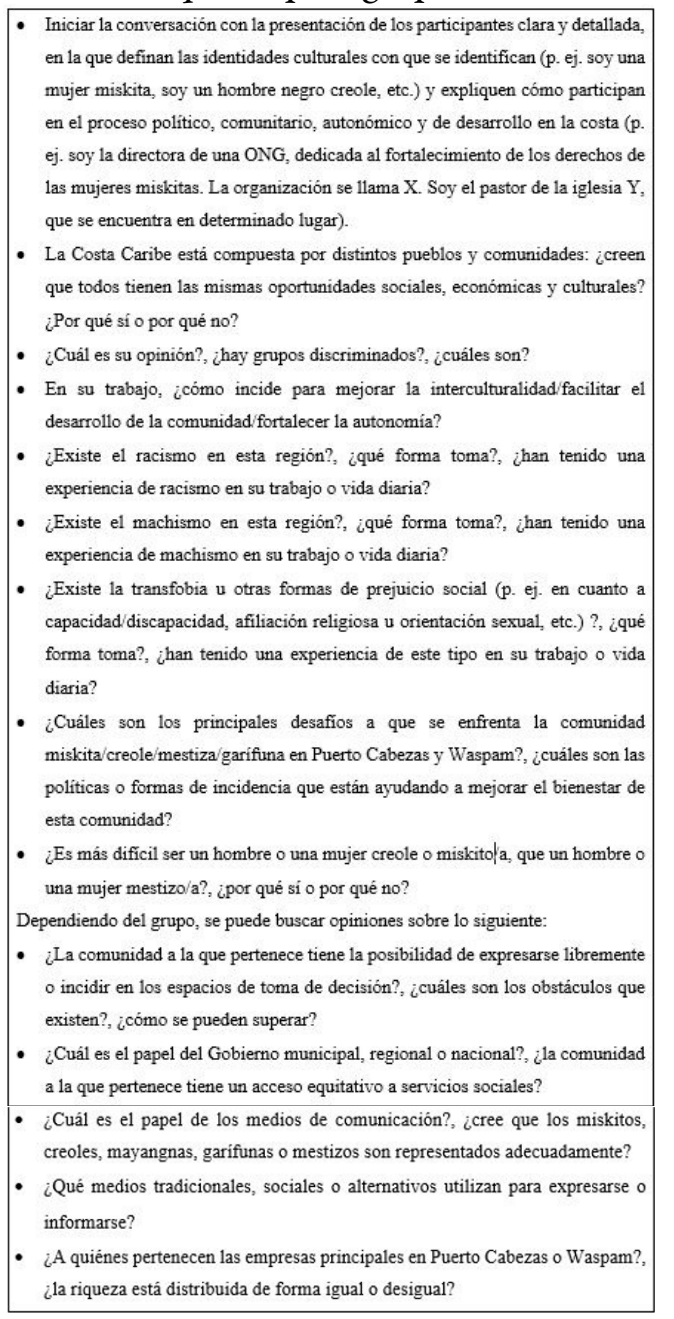

Fuente: Elaboración propia

A finales de octubre de 2016, divulgamos los resultados y análisis preliminares de los grupos focales en dos talleres, uno en Bilwi y otro en Managua. En Bilwi, compartimos nuestros resultados con los actores que participaron en los grupos focales y otras personas interesadas. En Managua, el espacio de socialización contó con la participación de académicos, medios de comunicación y miembros de ONG nacionales e internacionales, que tienen proyectos de desarrollo en la región, para que puedan utilizarlos en sus propias iniciativas. Posteriormente, elaboramos el primer borrador del artículo y lo compartimos con los participantes del estudio, con la invitación a nuevas contribuciones y reflexiones. Más adelante, incorporamos estos aportes en la versión final del texto. 


\section{Cuentas éticas y teóricas}

Es importante reconocer que tratamos temas de enorme complejidad teórica y ética. Consideramos al menos cuatro premisas éticas-teóricas: primero, las limitaciones teóricas asociadas a las identidades culturales; segundo, las relaciones históricas de poder en Nicaragua que siguen teniendo efectos en la actualidad; tercero, nuestras posicionalidades como investigadores; y cuarto, la contextualización del estudio dentro de la llamada “agenda del impacto", que domina el entorno de investigación británico en este momento.

Las identidades culturales son social y culturalmente construidas, no son ni esenciales ni biológicamente determinadas. Por lo tanto, tampoco pueden verse como entidades fijas. El género tiende a definirse de forma binaria, por ejemplo, hombre/mujer, pero esto también es una construcción, y en Latinoamérica puede considerarse un legado colonial (Lugones, 2010). La identidad de género va mucho más allá de estas categorías binarias, hay identidades trans, no binarias, tercer género, genderqueer etc., que están fuera de la cisnormatividad y que tenemos que tomar en cuenta. En el mismo sentido, tenemos claro que las razas biológicas no existen, pero el racismo sí, entonces los grupos racializados por el sistema colonial necesitan ocupar identidades positivas, identidades que contribuyan al desmantelamiento de las actitudes discriminatorias. Esto significa que las teorías sobre las identidades culturales que son dominantes en el pensamiento contemporáneo occidental no sirven para comprender las luchas descoloniales en Nicaragua. Siguiendo a Frantz Fanon, Ramón Grosfoguel (2018) distingue entre la llamada zona del ser, donde hay opresiones de clase o de género, pero los oprimidos gozan de privilegios raciales y la zona de no ser, donde la humanidad de los oprimidos no es reconocida o donde sus conocimientos o formas de pensar han sido inferiorizados. Entonces, en la zona del ser, el antiesencialismo sirve para desinflar identidades exageradas, mientras que en la zona del no ser [...] el antiesencialismo radical se convierte en un método colonial porque en la zona del no ser se trata de identidades, de epistemologías, de espiritualidades, de formas de ser y de existir que no han sido exageradas - o infladas - por las relaciones coloniales sino, al contrario, han sido desinfladas, inferiorizadas, invisibilizadas, destruidas por la colonialidad. Entonces, ahí hay un proceso de reconstrucción necesario que no se puede dar desde una perspectiva antiidentitaria radical o antiesencialista radical porque en la medida en que apliques ese tipo de antiidentitarismo radical o antiesencialismo radical no le permites a esos pueblos reconstruir sus identidades, sus saberes, sus conocimientos, sus formas de ser, de vivir, sus espiritualidades, etc. (Grosfoguel, citado en Ruiz Trejo, 2013)

Para entender la condición colonial de Nicaragua, no se pueden perder de vista los aportes del feminismo descolonial y del trabajo crítico sobre el desarrollo. Por lo tanto, reconocemos que las categorías identitarias que utilizamos en este artículo - género, mestizaje, costeños - son problemáticas porque surgen en parte del pensamiento colonial y eurocéntrico y, posiblemente, sugieren una homogeneidad, que no existe. Los mestizos del Pacífico, por ejemplo, son fragmentados por múltiples identidades de clase, género, sexualidad y edad en consecuencia, el cuestionamiento y la deconstrucción de estas categorías es parte importante de un análisis decolonial e interseccional. Desde este punto de vista, comprendemos el mestizaje no como una serie de categorías esenciales que se aplican a los que se identifican como nicaragüenses mestizos, los cuales también están negociando la colonialidad de forma heterogénea e interseccional, sino como un discurso hegemónico, que no solo tiene "un carácter excluyente", sino también "genérico y sexual" (Mendoza, 2001, pp. 257, 259), y como una ideología estatal para manejar las diferencias étnicas (Cunin y Hoffman, 2013). Se trata de un discurso que ha creado un imaginario nacional racista y androcéntrico, ya que plantea la presencia de negros, sobre todo, e indígenas como un obstáculo para la construcción y el desarrollo de la nación y entiende las identidades raciales de forma jerárquica. Para Breny Mendoza (2001, p. 267), quien profundiza en la situación de Honduras, el mestizaje borra la presencia negra, expropia la historia indígena, y complica "nuestra comprensión de las relaciones raciales". En el caso de Nicaragua, el mestizaje como discurso e ideología sirve para restringir las formas de inclusión facilitadas por la autonomía (González, 2013). A pesar 
de que el mestizaje se moviliza de forma dinámica y contradictoria en las prácticas diarias e institucionales, en ocasiones puede desarticularse de sus amarres eurocéntricos y destinarse a fines descoloniales.

El ser miskito o creole es una condición cultural que está arraigada en historias y geografías complejas que se han desarrollado en la zona del no ser. Significa que las construcciones raciales o de género tienen efectos materiales importantes tanto negativos como positivos. Negativos, porque el racismo y el machismo persisten a veces de forma muy violenta y constituyen obstáculos importantes a la dignidad humana, y positivos, porque son fuentes de orgullo, pertenencia cultural y conocimientos que empoderan. Algunas formas de ser culturales se crearon como resultado del colonialismo y el desarraigo forzado de muchas personas de descendencia africana que tuvieron que sobrevivir en condiciones difíciles y en el proceso crearon culturas vibrantes. Entonces, el fortalecimiento de la identidad colectiva como Creoles, Miskitos o mayangnas es un mecanismo importante en contra del racismo y una forma de valorar lo que ha sido invisibilizado por el sistema colonial. Los indígenas y los afrodescendientes de la Costa Caribe tienen que emprender un proceso de reconstrucción de identidades, que inicia con el reconocimiento de formas de pensar y cosmovisiones no eurocentradas

Entonces, el desmantelamiento del racismo en Nicaragua es un proceso también epistemológico y significa hacer mucho más que celebrar la diversidad cultural del país. El discurso de la diversidad tiene muchos límites en cuanto a la búsqueda de la descolonización. Como han señalado algunas escritoras indígenas y afrodescendientes, como Silvia Rivera Cusicanqui de Bolivia y Juliet Hooker (2005a), intelectual creole del Caribe sur de Nicaragua, el multiculturalismo oficial y la movilización del discurso de la diversidad son las formas más eficaces que tiene el Estado mestizo hegemónico para neutralizar las reivindicaciones históricas de los grupos marginados. Para Rivera Cusicanqui, la celebración de la diversidad étnica por la clase política latinoamericana es un mecanismo para ocultar nuevas formas de colonización. Hay que celebrar la diversidad cultural de Nicaragua, pero no utilizar esa narrativa para acabar con las reivindicaciones de índole política.

Otro limitante ético y teórico del presente estudio es que lograr la representatividad de todos los grupos culturales que ocupan espacios de poder en la costa aunque es necesaria, es, al mismo tiempo, casi imposible. Esta imposibilidad se debe al hecho de que las identidades étnicas y de género no existen aisladas de otras, como la clase social, la adscripción partidaria o la afiliación religiosa. Entonces, no es posible decir que un hombre miskito pueda representar a todos los hombres Miskitos o que una mujer creole pueda representar a todas las mujeres Creoles, debido a que otras identidades pueden ser más significativas. Si esta persona privilegia intereses partidarios, masculinos o urbanos sobre otros intereses culturales, la representación posible se limita. Lo anterior, muestra que los miembros de un determinado grupo cultural no son homogéneos, y en un determinado momento una faceta de su identidad podría ser privilegiada sobre otras facetas. Por lo tanto, es posible que una mujer creole con enfoque feminista pueda servir mejor a las mujeres miskitas que un hombre miskito que lucha por los intereses de los Miskitos, pero, al mismo tiempo, demuestra una actitud machista o derogatoria hacia las mujeres.

Además, existen relaciones históricas de poder que tenemos que tomar en cuenta. Estas relaciones se construyeron en el pasado - y tienen mucho que ver con la forma en que la Costa Caribe se incorporó en la nación-, pero siguen vigentes en la actualidad. Nicaragua se destaca por su abundante riqueza cultural y lingüística, que está amenazada por varios procesos, no solo la globalización, en general, sino también procesos a escala nacional, como la degradación ambiental y la mestización de la región, provocados por los intensos flujos migratorios del Pacífico y el avance en la frontera agrícola hacia los territorios comunitarios y los últimos vestigios de bosques naturales en el país. En ese sentido, un programa para rectificar formas históricas y contemporáneas de exclusión debe tener mecanismos que prioricen a los grupos marginados, a corto o largo plazo. Una comunidad donde todos tienen la capacidad de participar en los procesos de toma de decisión será mejor para todos. No se trata de un juego de suma cero, se trata de restituir derechos que han sido negados y, a la vez, proteger y revitalizar conocimientos y prácticas culturales que está en riesgo de desaparecerse. Emprendemos este trabajo teniendo en cuenta la existencia persistente de pautas coloniales 
que estigmatizan y de una memoria colectiva dolorosa, sobre todo dada la forma violenta mediante la cual la Costa Caribe llegó a ser parte de la nación nicaragüense. Simultáneamente, reconocemos que la posición social asumida y atribuida no se desmantela fácilmente.

Otra consideración ética es el hecho de que nuestro estudio emerge y se contextualiza dentro de la agenda de impacto difundida por los consejos británicos de investigación, bajo un gobierno conservador y neoliberal. Notamos que esta agenda que se ha establecido en los últimos años es muy controvertida y ha llevado a debates sustanciales sobre sus méritos y sus desventajas. Si bien la agenda puede ser vista como la instrumentalización y la neoliberalización de los proyectos de investigación (Slater, 2012), esos resultados no son inevitables dada la maleabilidad y la falta de definición clara del concepto (Pain et al., 2011; North, 2013; Rogers et al., 2014). De hecho, así como el concepto de desarrollo ha sido transformado y subvertido en la práctica diaria por los actores de base, y se ha adaptado no para implementar, sino para cuestionar e invertir las estrategias exógenas (Cupples et al., 2007); también el concepto de impacto puede adaptarse a diversas situaciones en las que el neoliberalismo, el racismo y la degradación ambiental están causando grandes daños para una población local. Además, nuestro proyecto se lleva a cabo en colaboración con Uraccan, una universidad comunitaria intercultural, cuya visión se basa en el acompañamiento de "procesos de gestión e incidencia, para la construcción de ciudadanías interculturales de género, que conlleven al buen vivir y la autonomía de los pueblos indígenas, afrodescendientes, mestizos y otras comunidades étnicas" (Uraccan, www.uraccan.edu.ni/content/visión-y-misión). Entonces, los programas de estudio y las investigaciones que emprenden los profesores y estudiantes de Uraccan están diseñados para mejorar la calidad de vida de las comunidades de la región y, por lo tanto, ya están arraigados en una noción culturalmente específica de impacto (Cupples y Glynn, 2014). Podríamos decir que Uraccan tiene su propia agenda de impacto descolonial.

\section{La coyuntura}

Para hacer un trabajo de este tipo es muy importante fijarse en los elementos coyunturales que ayudan a crear, mantener o desmantelar las barreras de la participación equitativa. Tenemos que ser conscientes del contexto cultural, político y económico específico en que se trabaja. Esta sección describe estos elementos superpuestos e interrelacionados de forma más detallada.

El desarrollo de la Costa Caribe norte se ve impedido por la falta de inversión en la infraestructura. En este lado de la costa, las condiciones son mucho peores que las del Pacífico. Se evidencia el ingreso de alguna inversión estatal, la cual puede traer efectos positivos, pero, por ahora, hay insuficiente acceso al agua potable, la energía, la salud, la educación y el transporte. Además, algunos costeños se quejan de que aunque hay señales de inversión en infraestructura, los proyectos se inician sin consulta con las comunidades.

No hay duda de que la pobreza y la desigualdad constituyen unos obstáculos muy grandes para la participación equitativa. La Costa Caribe tiene altos e inaceptables niveles de pobreza, los cuales son mucho más altos que los promedios nacionales, a pesar de ser una región muy rica en recursos naturales y estratégicos en madera, pesca y minería. Estos elementos estructurales empeoran debido a factores de índole ambiental y flujos migratorios, tanto entrantes como salientes. Además de la destrucción ambiental causada por la sobreexplotación de estos recursos durante las últimas dos décadas, los medios de subsistencia y las culturas costeños han sido amenazados por intensas presiones migratorias del Pacífico, proceso que los costeños denominan "invasión del Pacífico". La degradación ambiental se debe a factores tanto locales como globales. Es decir, los efectos del despale, la sobreexplotación de la langosta, la ganadería intensiva, y la presencia de los colonos se han exacerbado con el cambio climático y el hecho de que las causas del cambio climático se localizan en el Norte global, donde las emisiones de carbón son muy altas, mientras que los efectos nefastos se sienten en Nicaragua y en otras partes del Sur global. Asimismo, los agricultores mestizos de la región del 
Pacífico, conocidos como colonos o terceros, se han establecido en tierras comunales y se dedican a diversos modos de agricultura, la ganadería, la tala de árboles y la compraventa ilegal de tierras ancestrales, lo que da lugar a conflictos sustanciales y a la destrucción de recursos graves. Muchos costeños indígenas han sido asesinados, secuestrados o heridos por colonos cada vez más violentos (Cupples y Glynn, 2018).

Con respecto a la migración, es importante destacar que la región pierde mucho talento, ya que muchos costeños salen de esta en busca de oportunidades económicas en Managua, Costa Rica y Estados Unidos. Debido a la falta de fuentes de empleo digno, muchos de los costeños más talentosos abandonan la región en busca de trabajo y un mayor nivel de vida. Hay muchos costeños Creoles que por su dominio del idioma inglés terminan trabajando en los call centers en Managua. Este fenómeno empeora el declive de las lenguas de la región, sobre todo del inglés creole, que se ve amenazado por el creciente uso del español en la región. El miskitu, por tener mayor número de hablantes, es más resiliente.

Los elementos coyunturales de índole política y cultural también son de importancia. La Costa Caribe por ser una región multiétnica y multicultural donde coexisten diversas lenguas, prácticas y espiritualidades, contiene mucha diversidad cultural, que constituye una fuente de riqueza. Aunque esta diversidad se celebra, a veces de forma folklórica, el racismo y el machismo siguen siendo persistentes. Los costeños negros e indígenas siguen siendo objeto de prácticas discriminatorias en las instituciones, los medios de comunicación y la vida diaria. En Nicaragua, existe el fenómeno que Quince Duncan (2012) llama endophobia o la ideología del mestizaje, es decir, la idea de que las culturas e identidades indígenas y afrodescendientes son un obstáculo para el desarrollo nacional y para la construcción de la nación (Hooker, 2005b). Estas actitudes culturales persisten en una época de creciente autoritarismo en Nicaragua, en la que vemos el cierre de los espacios democráticos para la sociedad civil y el aumento de corrupción política cada vez más endémica, incluso en el tráfico ilegal de tierras ancestrales. Muchos ciudadanos nicaragüenses, por temor a represalias, no expresan su voz opositora ni condenan el control, a veces exagerado, que tienen los partidos políticos sobre la participación política. La libertad de prensa está siendo seriamente restringida a través de una serie de medidas, y se hace cada vez más difícil practicar un periodismo independiente o comunitario (Cupples y Glynn, 2018). En este contexto, los espacios de debate y reflexión, que son necesarios para la democratización y el fortalecimiento de la autonomía, desaparecen. La desdemocratización significa que hay una tendencia a hablar de la autonomía entre comillas, porque es un proceso que sufre muchos retrocesos. Muchos costeños afirman que no hay autonomía verdadera porque los recursos naturales de la región como la pesca y la madera no están en manos de los pueblos costeños. Sin embargo, se debe reconocer que mediante las Leyes 28 y 445 las identidades culturales se han convertido también en identidades jurídicas, lo que muestra sus potencialidades (Watts, 2003).

\section{Los resultados}

Aunque documentar la distribución del poder es importante, es una tarea limitada en términos de las dificultades para lograr una comprensión profunda sobre las barreras de la participación. En cuanto a las posiciones políticas por elección popular, como concejales, es bastante fácil contar la cantidad de indígenas, Creoles, hombres y mujeres. Por su parte, resulta más complicado el análisis de las organizaciones de la sociedad civil o los medios de comunicación, donde trabajan muchas personas distintas con capacidades de toma de decisión que no se pueden medir. En términos cuantitativos, en general, hay una situación bastante optimista (tablas $2,3,4$, y 5$)^{3}$. Se nota que hay representatividad de las mujeres y de todos los grupos indígenas $\mathrm{y}$ afrodescendientes que habitan la costa. En algunos espacios, los Miskitos tienen mayor presencia que los mayagnas y los Creoles. Notamos, además, que las iglesias costeñas se han indigenizado o creolizado por completo, aunque predominan los hombres en las posiciones de liderazgo. 
TABLA 2

Datos cuantitativos regionales

\begin{tabular}{|l|}
\hline Presidentes de las comisiones del Consejo Regional \\
\hline Total: 16 \\
\hline 8 mujeres, 8 hombres. \\
\hline 9 miskitos, 4 mestizos, 2 creoles, 1 mayangna. \\
\hline Miembros de la Junta Directiva del Consejo Regional \\
\hline Total: 6 \\
\hline 2 mujeres, 4 hombres. \\
\hline 2 mestizos, 2 miskitos, 1 mayangna, 1 creole. \\
\hline Concejales regionales de la Costa Caribe norte \\
\hline Total: 48. \\
\hline 23 hombres, 25 mujeres. \\
\hline 22 miskitos, 20 mestizos, 4 creoles, 2 mayagnas. \\
\hline
\end{tabular}

Fuente: Elaboración propia

TABLA 3

Datos cuantitativos municipales, Waspam

\begin{tabular}{|l|}
\hline Presidentes de comisiones del Consejo Municipal de Waspam \\
\hline Total: 5 . \\
\hline 3 mujeres, 2 hombres. \\
\hline 5 miskitos. \\
\hline Miembros de la Junta Directiva Municipal de Waspam \\
\hline Total: 3 . \\
\hline 1 mujer, 2 hombres. \\
\hline 3 miskitos. \\
\hline
\end{tabular}

Fuente: Elaboración propia

TABLA 4

Datos cuantitativos municipales, Bilwi/Puerto Cabezas

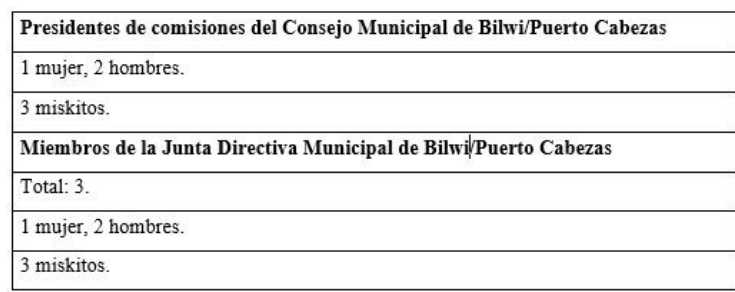

Fuente: Elaboración propia

TABLA 5

Datos cuantitativos territoriales

\begin{tabular}{|l|}
\hline Presidentes territoriales del municipio de Bilwi/Puerto Cabezas \\
\hline Total: 5 . \\
\hline 1 mujer, 4 hombres. \\
\hline 5 miskitos. \\
\hline Presidentes territoriales del municipio de Waspam \\
\hline Total 5. \\
\hline 2 mujeres, 3 hombres. \\
\hline 5 miskitos. \\
\hline
\end{tabular}

Fuente: Elaboración propia

Como suele ocurrir con muchos estudios, son los datos cualitativos los que proporcionan información más matizada, detallada y rica. Los siguientes hallazgos son los temas más destacados de los grupos focales, agrupados según temática. En primer lugar, los grupos focales han enfatizado que hay un sentimiento generalizado de discriminación y exclusión entre los pueblos indígenas y afrodescendientes de la Costa Caribe. 
Aunque hay señales de participación, la situación sigue siendo muy negativa. En particular, los participantes hablaron de la falta de respeto para los gobiernos territoriales, y notan que circulan discursos discriminatorios de juzgamiento y que son más fuertes en tiempos de crisis al echar la culpa a los que son distintos. Relacionado con este sentimiento, se evidencia que no hay igualdad de oportunidades para cada uno de los grupos culturales.

Todos los grupos se sienten marginados, pero hay más movilidad social entre algunos debido a factores coyunturales muy específicos. Los participantes reconocen la necesidad de participar, pero a veces, el sentimiento de inferioridad y los estereotipos se internalizan de forma problemática, y para algunos constituyen barreras culturales insuperables. Esta internalización lleva a que, en ocasiones, las mujeres mismas sean quienes reproducen el machismo, o que los grupos culturales propaguen estereotipos discriminatorios generados en otros lugares. La inferiorización de las identidades y epistemologías costeñas por la cultura dominante resulta en una autoestima baja en algunas personas, quienes no tienen confianza para participar o acceder a los espacios de toma de decisión. A veces las mismas lenguas indígenas, que deberían ser fuente de orgullo, se convierten en fuente de vergüenza. El buen dominio del español puede dar lugar a oportunidades económicas o profesionales, pero esta movilidad social podría producirse a Costa de la supervivencia o revitalización cultural. Además, la participación por sí sola no garantiza la transformación social.

Otro tema importante fue el lugar que se asigna a las mujeres en los espacios de participación: muchas veces, aunque las mujeres están presentes en los espacios de toma de decisión, no se les reconoce el derecho a decidir, debido al machismo arraigado. Las formas de discriminación que existen son difíciles de desmantelar porque se manifiestan de forma interseccional. Es decir, el racismo existe, pero muchas veces se ve agravado por la pobreza, el machismo, la homofobia o la transfobia. Las identidades étnicas se entremezclan y no se pueden separar de las identidades de género, clase y orientación sexual. Es mucho más difícil superar el racismo como persona indígena o afrodescendiente, si se es pobre o transexual.

En el caso del machismo, su transformación requiere de un conjunto de estrategias diferenciadas. Los participantes afirmaron que este existe entre todas las culturas de la costa, pero se manifiesta de distintas maneras. En las culturas miskitas se encuentran formas matriarcales, que no hay en las culturas mestizas. Por lo tanto, es necesario buscar cómo fortalecer estas formas matriarcales y utilizarlas en la lucha por la igualdad de género.

Algunos de los participantes se mostraron perturbados por la fluidez de la identidad étnica. Aunque reconocen en las palabras de una participante que "las etnias puras no existen", nos contaron, por ejemplo, que hay Creoles que se identifican como Miskitos o Miskitos que se identifican como Creoles en busca de supervivencia económica, para ganar acceso a los proyectos de desarrollo o por razones políticas. Una participante lo expresó así:

¿Cuánta representatividad existe a nivel de los gobiernos regionales? Y también cuestionarnos por qué ni siquiera estamos representados, ¿por qué una persona dice ser negra o creole?, ¿qué representa eso? Hace que se pierda incluso la identidad de los negros representados en la Costa Caribe norte. (Grupo focal, Bilwi, septiembre del 2016)

Lo anterior, muestra que algunas personas adoptan identidades alternativas para obtener algún tipo de beneficio. Aunque es posible que los cambios identitarios sean realizados deshonestamente, es muy importante reconocerlos, ya que las identidades son fluidas sobre todo en una región donde ha habido muchas formas de mezcla cultural durante muchos años, y donde la pertenencia cultural puede ser múltiple.

Una participante en el taller de Managua refirió la Declaración de las Naciones Unidas sobre los Pueblos Indígenas, en su artículo 33, que afirma que los indígenas son libres de escoger y determinar su propia identidad. Esta participante tiene una madre creole y un padre miskito y dijo que, aunque ella se autodefine como creole, sus hermanas han privilegiado sus identidades miskitas. De todos modos, tanto la cultura miskita como la creole influyen sobre su visión del mundo y sus prácticas culturales cotidianas. Por lo tanto, no debería 
sorprender cuando una persona que hemos identificado como perteneciente a un grupo cultural costeño, se identifica con otro.

Los participantes también llamaron nuestra atención a las diferencias que existen entre las zonas rurales y urbanas. Creen que las ciudades ofrecen más posibilidades de superar la marginación que las zonas rurales. Las mujeres sufren el machismo en toda la región, pero las rurales sufren más que las que viven en zonas urbanas. Los mayangnas se encuentran excluidos por ser un pueblo indígena y una minoría, pero también porque la población mayangna es principalmente una población rural. Los Creoles, por ser un grupo mucho más urbanizado, tienen algunas ventajas en cuanto al acceso a las oportunidades.

Todos los participantes valoran con creces el marco legal autónomo, que ha aumentado las oportunidades para los grupos culturales costeños y ha asegurado los derechos territoriales, pero notan que este se ciñe a un marco jurídico eurocéntrico. Por ejemplo, la Ley de Autonomía reconoce los derechos ancestrales, pero coexiste con la Ley Electoral y un sistema de gobierno importados de países europeos. Además, la existencia de un sistema de gobierno eurocéntrico se agudiza con la injerencia excesiva de los partidos políticos, sobre todo del partido de gobierno, en los espacios de poder. Con esto, se evidencia que tanto el creciente autoritarismo como el modelo político eurocéntrico, que está en vigor, socavan la lucha por la autonomía. Los participantes mencionaron que sería muy bueno si las comunidades pudieran elegir a sus líderes como lo han hecho tradicionalmente, sin ninguna intervención de los partidos políticos. Adicionalmente, enfatizaron en que la región tiene mucha riqueza cultural que no se contempla en los procesos de búsqueda de soluciones. Esta riqueza incluye las formas de organización ancestrales, la tenencia comunal y no capitalista de la tierra, la forma recíproca de organizar el trabajo agrícola, y la apertura al sincretismo religioso. Todos estos elementos son conocimientos y recursos muy valiosos, que se pueden utilizar para combatir la discriminación y aumentar la participación en los espacios de poder.

Los grupos focales mostraron la necesidad de incrementar la inversión estatal en la región. Declararon que la inversión infraestructural estatal es inadecuada o se hace al margen de las comunidades, sin consulta apropiada. Aunque los participantes se alegran de que el agua, la energía, la salud y la carretera se hayan priorizado en el Plan de Desarrollo, se identifica fricción por la insuficiente negociación por parte del Estado con las comunidades afectadas. El hecho de que los recursos naturales de la región y la fuente principal de riqueza sigan concentrados y en manos ajenas reduce bastante la efectividad de la Ley de Autonomía. Los costeños continúan sin fuentes de empleo digno; muchas personas, como los buzos Miskitos se han quedado sin trabajo como consecuencia de sus condiciones de trabajo precarias y riesgosas. Si bien hay algunas mejoras en el acceso a la educación, los participantes lamentan la falta de inversión en el rescate lingüístico y en la educación bilingüe.

La Iglesia latinoamericana ha jugado un papel contradictorio en cuanto a los derechos de los indígenas y las mujeres, y a los procesos de liberación. Durante el último siglo y medio, se han dado relaciones tensas entre las principales iglesias de la costa, como la morava y la católica, y las cosmovisiones indígena y creole. A veces, los líderes religiosos, a pesar de ser indígenas o afrodescendientes, se han opuesto a algunas creencias miskitas o Creoles. Durante la guerra de los años ochenta, la Iglesia morava se convirtió en firme defensora de los derechos indígenas y, en años más recientes, ha habido una paulatina y cautelosa convergencia entre la cosmovisión cristiana y la cosmovisión miskita. Los participantes afirmaron que, por lo general, las iglesias costeñas y las emisoras cristianas hacen un aporte progresista e intercultural —y no fundamentalista como en otras partes del mundo- a los procesos de desarrollo. Estas instituciones tienen un papel importante en los procesos de paz, forman comisiones para acabar con los conflictos, pero podrían abrirse más a las identidades alternativas en cuanto al género y la orientación sexual. En síntesis, todas las religiones de la costa presentan tendencias contradictorias, pero se nota que la flexibilidad teológica es una fuerza y necesidad importante.

Aunque la mayoría de los hallazgos revelan un panorama negativo, una cosa muy positiva que surgió en los grupos focales es que los costeños se han apropiado del tema de la interculturalidad y hay intentos verdaderos 
de hacerla efectiva. Nadie quiere que sea un discurso vacío, sino que se cumpla de forma material y visible en la vida diaria.

\section{Propuestas y recomendaciones}

Con base en los hallazgos, planteamos una serie de propuestas que esperamos sean útiles para todos los actores interesados en fortalecer el desarrollo en la Costa Caribe. Creemos que tenemos que: 1) matizar la cuestión de la proporcionalidad de la representatividad;2) descolonizar la legislación, las propuestas de desarrollo y los proyectos de investigación; 3) iniciar un programa de sensibilización en el Pacífico; 4) detener los procesos de desdemocratización, e 5) iniciar un programa de revitalización de lenguas. A continuación, tratamos cada una.

1. Repensar la proporcionalidad. Consideramos que la proporcionalidad por sí sola no es la manera más apta para lograr la participación equitativa de todos los grupos culturales costeños. Los mestizos son el grupo más grande y el que más crece, pero su cultura no se enfrenta a las mismas amenazas que los grupos indígenas o afrodescendientes. La proporcionalidad no ayuda a deconstruir la ideología del mestizaje. Por lo tanto, los grupos minoritarios y los más discriminados requiere más representación que los grupos dominantes para que sus perspectivas y sus voces lleguen a formar parte de los debates nacionales. Este es un mecanismo débil para lograr la representatividad porque las identidades étnicas se entremezclan con otras identidades, lo cual implica una gran diversidad, y porque muchas veces los líderes priorizan identidades partidarias. Sin embargo, creemos que un análisis de este tipo tiene que distinguir entre los mestizos del Pacífico y los mestizos costeños, ya que estos últimos tiene una vinculación mucho más fuerte con la región.

2. Fortalecer la descolonización. Es urgente descolonizar la legislación, las propuestas de desarrollo y los proyectos de investigación en Nicaragua. Estos tienen que ser descoloniales y planteados de abajo hacia arriba, es decir, desde la participación ciudadana. Es necesario reconocer que el concepto de desarrollo fue inventado por los poderes del Norte global después de la Segunda Guerra Mundial como estrategia geopolítica (Escobar, 1995). Aunque representa un tema muy cuestionado, puede ser apropiado o rearticulado por los actores de base. Lo anterior, muestra que es posible alcanzar un desarrollo indigenizado o africanizado. Todos los participantes estuvieron a favor de la creación de planes de desarrollo construidos de abajo hacia arriba por los grupos culturales, con una alta participación de los jóvenes. Afirmaron que el desarrollo debe partir de las comunidades mismas para que no resulte en transculturalización. Una participante lo expresó así:

Me parece muy acertado el hecho de que nos cuestionemos qué implica el desarrollo para los costeños porque también estamos acostumbrados a asumir conceptos, los cuales no están necesariamente acordes a nuestra realidad y a nuestros conceptos. Tenemos como lógicas paralelas que vienen desde arriba hacia abajo, y nuestra cultura ancestral que te dice que la lógica es invertida, es de abajo hacia arriba, lo que piensa la comunidad, y eso te permite construir varios aspectos. (Presentación del proyecto Bilwi Puerto Cabezas, octubre 2016)

Las agencias gubernamentales y no gubernamentales tienen que reconocer que una intervención vertical, que no toma en cuenta la cultura ancestral y no adopta una perspectiva interseccional, puede traer más perjuicios que beneficios.

Asimismo, es sumamente necesario que los conocimientos y las prácticas indígenas y afrodescendientes se tomen en cuenta en el momento de crear una nueva legislación e invertir en proyectos de infraestructura. Durante los grupos focales, uno de los participantes se expresó en favor de la coinversión. En 2016, la Asamblea Nacional aprobó la Ley de Asociación de Inversión Público-Privada, la cual supone que las únicas economías que existen en Nicaragua son la privada y la estatal, no toma en cuenta de que también hay economías comunitarias. Si los legisladores nacionales fueran más conscientes de las realidades culturales de la costa, habrían creado una ley de inversión pública-privada-comunitaria. Precisamente, uno de los participantes subraya que la economía de la Costa Caribe está basada en cuatro modelos distintos, lo que 
muestra que hay una mezcla de sistema capitalista empresarial, comercial local, latifundista-parcelario y de autoconsumo o comunitario. Por lo tanto, resulta indispensable fortalecer los modelos económicos que producen la vida y superar los limitantes en este aspecto del capital y el Estado.

En cuanto a la investigación, se deben evitar las prácticas extractivistas. El modelo extractivista no solo se aplica a los recursos naturales, sino también a los proyectos de investigación. Entonces, tenemos que diseñar los programas desde las comunidades, incluir las voces excluidas, compartir hallazgos antes de publicar e incorporar los aportes comunitarios en la versión publicada. En la presentación de Bilwi, un participante nos dijo "Yo me siento retratado", y otro nos agradeció por compartir los hallazgos. Manifestó que muchos investigadores han desarrollado proyectos en la costa, pero casi siempre desaparecen después de sacar la información y los costeños nunca llegan a saber cómo fue escrito el estudio. Entonces, es muy importante que se mantenga el compromiso después de los procesos de recolección.

En relación con este asunto, animamos a otros investigadores a incluir también el sector religioso. En ocasiones, los estudios enfocados en temas políticos o sociales no prestan suficiente atención al papel de la religión en las luchas por la justicia social, debido a las subjetividades ateas de los investigadores. Uno de los pastores que participó nos agradeció por haberles incluido: "siempre nos marginan de estas cosas, solo nos llaman cuando hay una huelga y un tranque en la carretera”.

3. Fomentar la sensibilización en el Pacifico. Se evidencia que existe un discurso fuerte de interculturalidad en la Costa Caribe y es un tema que se discute mucho en los centros de educación, los medios de comunicación, los talleres de la sociedad civil y en la calle. Sin embargo, este es un discurso casi ausente en la parte pacífica de Nicaragua. Creemos que es urgente sensibilizar a los mestizos del Pacífico, incluyendo a los niños y los adultos, sobre el hecho de viven en un país multiétnico. Los mestizos del Pacífico tienen que comprender que los flujos migratorios entrantes y salientes pueden enriquecer a una región o puede tener efectos destructivos en la supervivencia cultural de los grupos que han sufrido el racismo estructural. La Costa Caribe es parte de Nicaragua, por lo tanto, no se puede prohibir a los mestizos del Pacifico crear un negocio o buscar un trabajo en ese lado del país, pero los que llegan a esa costa deberían de saber y entender que hay culturas y prácticas distintas. En ese sentido, resulta urgente reescribir la historia de Nicaragua, para reconocer la herida colectiva costeña dejada por la violencia de 1894. La interculturalidad como concepto y como práctica debería de ser tan fuerte en el Pacífico como lo es en el Caribe.

4. Revitalizar la democratización. La interculturalidad significa fortalecer los espacios autónomos no alineados. No hay duda de que la partidización de las luchas por reclamar los derechos históricos está haciendo muchísimo daño. En ese sentido, se requiere buscar cómo minimizar la injerencia de los partidos políticos en los asuntos comunales costeños. Es necesario abrir los espacios públicos y mediáticos donde se pueden debatir los procesos asociados a la autonomía y aprender de estos. Como dijo una participante, "tenemos que alfabetizarnos nosotros mismos". Teniendo en cuenta que la Ley de Autonomía se creó en tiempos de guerra, este es un buen momento para iniciar un diálogo regional y nacional sobre la autonomía. Se reconoce el trabajo importante de Uraccan en este aspecto, dado que ha creado varios espacios y foros donde el público puede participar en debates sobre el tema. Este debe ser una prioridad en las agendas y en los medios nacionales, no solo en la Costa Caribe, sino también en el Pacífico.

Por otra parte, se evidencia que tanto en el Caribe como en el Pacífico los líderes políticos se aferran al poder y no dan el paso a líderes nuevos ni a los jóvenes. La democratización también se fortalecería con cambios más frecuentes de liderazgo políticos. Se abrirían los espacios de toma de decisión a una mayor cantidad de voces y perspectivas. De todos modos, se requiere detener el proceso de desdemocratización en Nicaragua.

5. Iniciar un programa de revitalización de lenguas. Aunque las lenguas indígenas y el inglés creole todavía tienen un gran número de hablantes nativos, hay una tendencia cada vez mayor a privilegiar el uso del español, lo cual pone estas lenguas en riesgo. Las lenguas indígenas y el inglés creole deberían convertirse en asignaturas escolares y universitarias, tanto en el Pacífico como en el Caribe. Sobre este aspecto, destacamos el caso de Nueva Zelanda, país en el que todos los niños, indígenas y no indígenas, aprenden a hablar y pronunciar 
correctamente el idioma indígena de los Māori (te reo), y la lengua Māori se habla en todos los actos públicos en el país. En Nicaragua, los actos oficiales nacionales nunca reconocen el carácter multilingüístico del país, y los padres nicaragüenses más acomodados creen que sus hijos tienen que viajar a los Estados Unidos para aprender inglés. Se plantea la relevancia incluir clases en la lengua miskitu y mayangna en las escuelas primarias, animar a los medios nacionales y comunitarios a incluir programas en miskitu, mayangna e inglés creole y promocionar la idea de que el inglés se pueda aprender también en la Costa Caribe nicaragüense.

\section{Conclusiones}

El racismo y el machismo se generaron en la Costa Caribe de Nicaragua en condiciones de colonialidad y se encuentran arraigados en la cultura política y en los discursos cotidianos. Sin embargo, es posible emprender acciones concretas para mejorar la situación y que dependen de la voluntad política. Las posiciones descoloniales, que surgen de los marcos teóricos culturales, se pueden movilizar y se pueden implementar en prácticas cotidianas e institucionales.

Planteamos cinco sugerencias concretas, fundamentadas en las teorías críticas culturales que consideramos que sirven para mejorar la participación y disminuir las pautas coloniales y opresivas que quitan el potencial de los actores. Está claro que no es suficiente incluir a las personas que han sido excluidas, es necesario también reconocer sus conocimientos, escuchar sus perspectivas e incorporarlos en las decisiones que se toman. En 2018, la situación en Nicaragua se empeoró y la violencia del Estado hacia los sectores progresistas, incluso los de la Costa Caribe, se incrementó. Pero hay una lucha de base por la democratización, caracterizada por un deseo de no volver a cometer los errores del pasado y hay muchos activistas jóvenes que están batallando por un futuro interseccional, en el cual se privilegien las voces de los sectores excluidos por una revolución extractivista, machista y racista. Esperamos que las recomendaciones sugeridas sean parte de la nueva Nicaragua, que se está intentando construir.

\section{Referencias}

Almada Navarro, E. M. (2009). El análisis de actores: metodología para el análisis contextual en bibliotecología y estudios de la información. En F. F. Martínez Arellano, y J. J. Calva González (comps.), Memoria del XXVI Coloquio de Investigación Bibliotecológica y de la Información (pp.162-182). UNAM.

Baracco, L. (comp.) (2018). Indigenous struggles for autonomy: The caribbean coast of Nicaragua. Lexington Books.

Cunin, E. \& Hoffmann, O. (2013). Introduction to blackness and mestizaje in Mexico and Central America. En E. Cunin \& O. Hoffman (comps.), Blackness and mestizaje in Mexico and Central America (pp.xi-xix).: AfricaWorld Press.

Cupples, J. \& Glynn, K. (2014). Indigenizing and decolonizing higher education on Nicaragua's Atlantic Coast. SingaporeJournal of Tropical Geography, 35(1), 56-71. https://doi.org/10.1111/sjtg.12051

Cupples, J. \& Glynn, K. (2018). Shifting Nicaraguan mediascapes: Authoritarianism and the struggle for social justice. Springer.

Cupples, J., Glynn, K. \& Larios, I. (2007). Hybrid cultures of postdevelopment: The struggle for popular hegemony in rural Nicaragua. Annals of the Association of American Geographers, 97(4), 786-801. https://doi.org/10.111 $1 / \mathrm{j} .1467-8306.2007 .00583 . x$

Duncan, Q. (2012). El Pueblo afrodescendiente: diálogos con el abuelo juan bautista yayah. Palibrio.

Escobar, A. (1995). Encountering development: The making and unmaking of the third world. Princeton University Press. 
González, M. (2013). Indigenous, afro-descendant, and mestizo costen\#os: Limited inclusion in the autonomy regime of Nicaragua. En E. Cunin \& O. Hoffman (comps.), Blacknessand mestizaje in Mexico and Central America (pp. 1-34). AfricaWorld Press.

Gordon, E. T. (1998). Disparate diasporas: Identity and politics in an African Nicaraguan Community. University of Texas Press.

Grosfoguel, R. (2018). What is racism: Zone of being and zone of non-being in the work of Frantz Fanon and Boaventura de Sousa Santos. En J. Cupples \& R. Grosfoguel (comp.), Unsettling Eurocentrism in the Westernized University (pp. 264-273). Routledge.

Hooker, J. (2005a). Indigenous inclusion/black exclusion: Race, ethnicity and multicultural citizenship in Latin America. Journal of Latin American Studies, 37(2), 285-310. https://doi.org/10.1017/S0022216X05009016

Hooker, J. (2005b). "Beloved enemies": Race and official mestizo nationalism in Nicaragua. Latin American Research Review, 40(3), 14-39. https://doi.org/10.1353/lar.2005.0051

Lugones, M. (2010). Toward a decolonial feminism. Hypatia, 25(4), 742-759. https://doi.org/10.1111/j.1527-

Mendoza, B. (2001). Le desmitologización del mestizaje en Honduras. Mesoamérica, 42, 256-278.

North, P. (2013). Knowledge exchange, 'impact' and engagement: Exploring low-carbon urban transitions. The Geographical Journal, 179(3), 211-220. https://doi.org/10.1111/J.1475-4959.2012.00488.X

Pain, R., Kesby, M. y Askins, K. (2011). Geographies of impact: Power, participation and potential. Area, 43, 183-8. https://doi.org/10.1111/j.1475-4762.2010.00978.x

Pineda, B. (2006). Shipwrecked identities: Navigating race on Nicaragua's Mosquito Coast. Rutgers University Press.

Rogers, A., Bear, C., Hunt, M., Mills, S. \& Sandover, R. (2014). Intervention: The impact agenda and human geography in UK higher education. ACME: An International E-Journal for Critical Geographies, 13(1), 1-9. https://acme -journal.org/index.php/acme/article/view/984/838

Ruiz Trejo, M. (2013). ¿Cómo luchar decolonialmente? Entrevista con Ramón Grosfoguel. Diagonal 1, April. https: //www.diagonalperiodico.net/saberes/como-luchar-decolonialmente.html

Slater, T. (2012). Impacted geographers: a response to Pain, Kesby and Askins. Area, 44(1), 117-119. http://dx.doi. org/10.1111/j.1475-4762.2011.01067.x

Tapella, E. (2007). El mapeo de actores claves, documento de trabajo del proyecto Efectos de la biodiversidad funcional sobre procesos ecosistémicos, servicios ecosistémicos y sustentabilidad en las Américas: un abordaje interdisciplinario. Universidad Nacional de Córdoba, Inter-American Institute for Global Change Research (IAI).

Watts, M. (2003). Development and governmentality. Singapore Journal of Tropical Geography, 24(1), 6-34. http://d x.doi.org/10.1111/1467-9493.00140

\section{Notas}

* Artículo de investigación.

1 Para análisis detallados sobre estas relaciones históricas de poder, véase Pineda (2006), Gordon (1998), Baracco (2018).

2 Nuestras posicionalidades como investigadores también son relevantes. Los autores somos un equipo de cuatro personas, una extranjera, y tres nicaragüenses, solo uno de los cuales es de origen costeño. Nuestro trabajo fue posible gracias a la cooperación de desarrollo, la incidencia en la sociedad civil y la investigación académica en dos universidades, una en Nicaragua y otra en Escocia. A pesar de los orígenes ajenos de tres miembros del equipo, todos tenemos muchos años comprometidos con el trabajo, la colaboración entre nosotros y con las y los costeños, la divulgación académica sobre las realidades de la región, y la incidencia o mejoramiento del nivel de vida en la región. Nuestro compromiso con la región es inquebrantable y a largo plazo.

3 Estos datos fueron compilados antes de las elecciones municipales de 2017.

4 Al final de un libro basado en el trabajo etnográfico a largo plazo con poblaciones Creoles en el Caribe sur, Edmund T. Gordon (1998, pp. 262-263) describe las legítimas razones estratégicas y culturales por las que los creoles de la comunidad de Haulover cerca de la Laguna de Perlas adoptan identidades miskitas. 
Licencia Creative Commons CC BY 4.0

Cómo citar: Cupples, J., Incer Medina, G., Larios, I. y Lee Smith, D. (2020). Mapeo de actores en la Costa Caribe norte de Nicaragua: un análisis según el género y la identificación étnica y cultural. Universitas Humanistica, 89. https://doi.org/10.11144/Javeriana.uh89.macc 